\title{
Avaliação da função diastólica em crianças e adolescentes diabéticos tipo 1 - Existem sinais precoces de miocardiopatia diabética?
}

\author{
Assessment of diastolic function in children and \\ adolescents with type 1 diabetes mellitus - Are there \\ early signs of diabetic cardiomyopathy?
}

Claudia Duarte Santos ${ }^{1}$, Admar Moraes Souza ${ }^{2}$, Rosana Marques Pereira ${ }^{3}$, Margaret Cristina da Silva Boguszewski ${ }^{4}$, Suzana Nesi França ${ }^{4}$, Christopher Gallotti Vieira ${ }^{5}$, Marcos Furuta' ${ }^{6}$ Luiz de Lacerda Filho'

${ }^{1}$ Programa de Pós-graduação Saúde da Criança e do Adolescente Universidade Federal do Paraná (UFPR), Curitiba, PR, Brasil

2 Departamento de Clínica Médica, UFPR; Serviço da Ecocardiografia da Unidade de Métodos Cardiológicos, Hospital de Clínicas, UFPR, Curitiba, PR, Brasil ${ }^{3}$ Departamento de Pediatria,

UFPR; Unidade de Endocrinologia Pediátrica (UEP), Hospital de Clínicas, UFPR, Curitiba, PR, Brasil ${ }^{4}$ Departamento de Pediatria, UFPR, Curitiba, PR, Brasil

${ }^{5}$ Unidade de Endocrinologia Pediátrica (UEP), Hospital de Clínicas, UFPR, Curitiba, PR, Brasil ${ }^{6}$ Hospital de Clínicas, UFPR, Curitiba, PR, Brasi

Correspondência para: Claudia Duarte Santos Rua Petit Carneiro, 665, ap 62 80240-050 - Curitiba, PR, Brasil cds_duarte@yahoo.com

Recebido em 1/Dez/2010 Aceito em 26/Abr/2012

\section{RESUMO}

Objetivos: Avaliar a função diastólica (FD) de crianças e adolescentes diabéticos tipo 1 (DM1). Sujeitos e métodos: Estudo transversal de 67 DM1, sem comorbidades, e grupo controle ( $\mathrm{n}=$ 84) da mesma faixa etária. Analisaram-se: idade, sexo, índice de massa corpórea (IMC), Dopplerecocardiografia e eletrocardiograma de ambos os grupos e, nos portadores de DM1, o tempo de doença, $\mathrm{HbA1C}$, lipidograma e o valor da microalbuminúria. Resultados: Encontraram-se alterações diastólicas [( $A$ e $E$ mitral, relação $E / A$, tempo de relaxamento isovolumétrico (TRIV) e tempo de desaceleração da onda $E$ (TDE)] nos diabéticos, com maior prevalência nas meninas na faixa 13-17 anos. TRIV eTDE correlacionaram-se positivamente com o IMC $(p=0,028)$. Idade e tempo de doença foram fatores preditivos para a onda $A$ mitral $(p=0,004$ e 0,033, respectivamente). Conclusões: Alterações de FD foram detectadas nos DM1, com maior prevalência em meninas púberes. Tempo de doença e idade dos pacientes influenciaram parâmetros de FD. Arq Bras Endocrinol Metab. 2012;56(4):226-32

\section{Descritores}

Cardiomiopatia; diástole; diabetes melito tipo 1; criança; adolescente

\section{ABSTRACT}

Objectives: To evaluate diastolic function (DF) of children and adolescents with type 1 diabetes mellitus (DM1). Subjects and methods: Cross-sectional study of 67 otherwise healthy diabetic patients, and a control group $(n=84)$ in regard to age, sex, body mass index (BMI), Dopplerechocardiography, and ECG for both groups; and disease duration, $\mathrm{HbA1C}$, microalbuminuria, and serum lipids for DM 1 patients. Results: Diastolic alterations [(A and E mitral waves, E/A ratio, isovolumic relaxation time (IVRT) and E wave deceleration time (EWDT)] were found in diabetic patients, with higher prevalence among pubertal girls (13-17 years old). IVRT and EWDT correlated positively with BMI $(p=0.028)$. Chronological age and disease duration were predictive factors for mitral A wave ( $p=0.004$ and 0.033 , respectively). Conclusions: DF alterations were detected in the group of diabetic patients, with greater prevalence among pubertal girls; disease duration and age influenced parameters of DF. Arq Bras Endocrinol Metab. 2012;56(4):226-32

\section{Keywords}

Cardiomyopathy; diastole; diabetes mellitus, type 1; child; adolescent 


\section{INTRODUÇÃO}

$\mathrm{D}$ iabetes melito (DM) é um distúrbio metabólico de múltiplas etiologias, caracterizado por hiperglicemia crônica e distúrbios do metabolismo de carboidratos, gorduras e proteínas (1). A correlação entre DM e anormalidades cardiovasculares é significativa, pois hipertensão arterial sistêmica (HAS), doença coronariana (DC) e insuficiência cardíaca (IC) são responsáveis por $75 \%$ das mortes entre os diabéticos (2). Entretanto, uma nova entidade, a miocardiopatia diabética (MD), descrita pela primeira vez por Rubler e cols. (3), em 1972, tem sido responsabilizada por danos no miocárdio na ausência de HAS e DC. Atualmente, a MD é reconhecida como doença específica, que acomete 30\% dos pacientes com DMl (4).

A patogênese dessa miocardiopatia é desconhecida, porém, acredita-se que distúrbios metabólicos, como a hiperlipidemia, a hiperglicemia e o hiperinsulinismo na fase inicial do DM2 (5), além da microangiopatia, fibrose miocárdica e neuropatia diabética, possam participar da etiopatogenia da MD (4).

Técnicas não invasivas como a Dopplerecocardiografia podem identificar a presença precoce da $\mathrm{MD}$ $(6,7)$. Shapiro, em 1982 (6), descreveu a correlação entre alterações Dopplerecocardiográficas e microangiopatia em diabéticos.

Em 1986, Huikuri e cols. (8) reportaram disfunção sistólica em pacientes adultos portadores de diabetes tipo l submetidos ao teste de esforço. Raev (9), em 1994, também observou anormalidades da função cardíaca nesse grupo de pacientes, inicialmente do tipo diastólica, presentes em geral após oito anos de duração da doença, e a seguir do tipo sistólica, depois de quase duas décadas de enfermidade.

Com o propósito de investigar a função diastólica em diabéticos da faixa etária pediátrica, Hausdorf e cols. (10), em 1988, estudaram 36 crianças com DMl e relataram disfunção diastólica do ventrículo esquerdo (VE), avaliada pelo modo M. A presença e o grau da disfunção cardíaca tinham correlação significativa com o tempo de doença e controle metabólico. Por outro lado, Riggs e Transue (11), ao estudarem $20 \mathrm{DMl}$ normotensos, sem sinais de cardiopatia, entre 10 e 15 anos de idade, encontraram alterações da onda $\mathrm{E}$ mitral e relação $\mathrm{E} / \mathrm{A}$, porém, não observaram correlação dessas alterações com o tempo de doença e controle metabólico (hemoglobina glicosilada AlC).

Suys e cols. (12) estudaram crianças e adolescentes DMl e mostraram que disfunção diastólica do VE é mais prevalente em meninas adolescentes. Posteriormente, estudos com Doppler tecidual constataram que a disfunção cardíaca também ocorre no ventrículo direito (VD). Além dessas alterações, anormalidades sistólicas podem ser reconhecidas pelo Doppler tecidual na região do anel mitral (13).

Tendo em vista que a Dopplerecocardiografia é, atualmente, um método de investigação de lesões miocárdicas subclínicas, o presente estudo tem como objetivo avaliar a função diastólica em crianças e adolescentes portadores de DMl.

\section{SUJEITOS E MÉTODOS}

Foram avaliados 67 crianças e adolescentes (26 do sexo masculino e 41 do sexo feminino) com DMl seguidos regularmente na Unidade de Endocrinologia Pediátrica do Departamento de Pediatria do Hospital de Clínicas da Universidade Federal do Paraná. Todos os pacientes eram tratados apenas com insulina e não tinham comorbidades e/ou complicações diabéticas. O grupo controle foi composto por 84 crianças e adolescentes saudáveis (40 do sexo masculino e 44 do sexo feminino) pareados por idade. Para a medida do peso corporal (em quilogramas, $\mathrm{kg}$ ), utilizou-se balança da marca Filizola ${ }^{\circledR}$ (São Paulo, SP). Para a obtenção da estatura (em centímetros, $\mathrm{cm}$ ), utilizou-se estadiômetro fixo em parede (Stadiometer Mode S100, Ayrton Corporation ${ }^{\circledR}$, Prior Lake, Minesota). Esses parâmetros foram obtidos no dia do exame de Dopplerecocardiografia. A superfície corpórea (SC) e o IMC foram calculados pelas fórmulas: $0,0001 \times 71,84 \times$ (altura) $)^{0,725}$ e peso $/(\text { altura })^{2}$, respectivamente. $\mathrm{O}$ estádio puberal foi avaliado de acordo com Tanner (14).

Hemoglobina glicosilada AlC (HbAlC) foi medida pelo método HPLC, Kit comercial (BioRad) e aparelho BioRad D10 (BIO-RAD, Hercules, Califórnia, USA). A média dos valores das três últimas determinações foi considerada para cálculo estatístico. Os valores preconizados como adequados para crianças e adolescentes diabéticos foram: lactentes e pré-escolares $(0 \mathrm{a}$ 6 anos) $7,5 \%$ a $8,5 \%, 6$ a 12 anos $<8 \%$ e adolescentes e adultos jovens $<7,5 \%$ (15).

A dosagem da microalbuminúria foi realizada em urina de 24 horas, utilizando-se kit comercial da marca Abbott e o aparelho Architect ci 8200 - Abbott (Abbott, Mississauga, Ontário, Ca). Considerou-se presença de microalbuminúria quando a taxa de excreção urinária de albumina era entre 20 e $200 \mathrm{mcg} /$ minuto. No período do estudo, pelos protocolos da Unidade 
de Endocrinologia Pediátrica (UEP) baseados na American Diabetes Association (ADA), a dosagem da microalbuminúria era realizada apenas em pacientes com tempo de doença superior a cinco anos. Pela razão de a microalbuminúria ser um fator preditor para nefropatia e esta comprometer a função cardíaca, foram incluídos no estudo somente os portadores de DMl com pesquisa de microalbuminúria negativa.

Quanto ao tempo de doença, este não foi um fator de exclusão. A população avaliada foi composta de todos os portadores de DMl que preenchiam demais critérios de inclusão.

Colesterol total, HDL colesterol e triglicérides foram medidos pelo kit comercial (Abbott) pelo aparelho Architect c 8000 (Abbott, Mississauga, Ontário, Ca). O LDL foi calculado pela fórmula de Friedwald: Colesterol LDL $=$ Colesterol Total $-($ colesterol HDL + Triglicerídeos/5). A média dos valores das três últimas determinações foi considerada para cálculo estatístico. Embora pela ADA o valor de LDL desejável nas crianças diabéticas seja menor de $100 \mathrm{mg} / \mathrm{dL}$, os valores de referência considerados neste estudo foram os da Academia Americana de Pediatria para crianças acima de dois anos: colesterol total $<200 \mathrm{mg} / \mathrm{dL}, \mathrm{LDL}<130 \mathrm{mg} / \mathrm{dL}$, $\mathrm{HDL}>35 \mathrm{mg} / \mathrm{dL}$ e triglicérides $<150 \mathrm{mg} / \mathrm{dL}$ (16).

Este estudo foi autorizado pelo Comitê de Ética em Pesquisa do Hospital de Clínicas da Universidade Federal do Paraná. Os pais e/ou responsáveis foram informados sobre os objetivos do estudo e assinaram o Termo de Consentimento Livre e Esclarecido.

\section{Ecocardiografia}

Após a aferição dos dados antropométricos e da pressão arterial sistêmica com os pacientes sentados e em pé, realizou-se exame ecocardiográfico completo simultâneo com eletrocardiograma (ECG) na derivação D2. Foi utilizado o aparelho de ultrassom Philips Envisor (Philips Medical Systems; Bothell, Washington, USA) equipado com transdutor multifrequencial (2 a $4 \mathrm{MHz}) \mathrm{e}$ adaptado para Doppler tecidual. Os planos Dopplerecocardiográficos utilizados foram os preconizados pelo $\mathrm{Co}^{-}$ mitê da Sociedade Americana de Dopplerecocardiografia $(17,18)$. Os filtros do Doppler convencional e tecidual foram de 200 e 28, respectivamente, e ganho de 1,9. Para os valores de Doppler, foi utilizada a média das ondas de três ciclos cardíacos. A velocidade das ondas de Doppler foi gravada em velocidade de $100 \mathrm{~mm} / \mathrm{s}$ e realizada por um único operador, em um único momento.
Todos os exames Dopplerecocardiográficos foram armazenados em Compact Disc (CD).

A função diastólica foi avaliada pelas seguintes medidas: velocidades das ondas E e A (m/s) do fluxo mitral, relação $\mathrm{E} / \mathrm{A}$, tempo de desaceleração da onda $\mathrm{E}$ (TDE), tempo de relaxamento isovolumétrico (TRIV), fluxos pulmonares sistólico e diastólico, duração da onda "a" reversa da veia pulmonar (Ar) e Doppler tecidual no anel mitral porção septal. $\mathrm{O}$ índice de performance miocárdica (índice de TEI) do ventrículo esquerdo foi definido como a soma dos tempos de relaxamento e contração isovolumétricos dividida pelo tempo de ejeção. Esse índice foi utilizado para avaliação da função cardíaca global.

Para evitar a interferência da frequência cardíaca (FC) no cálculo da FD, utilizou-se o agrupamento por faixas etárias, segundo os critérios de O’Leary (19).

\section{Eletrocardiografia}

Os dados de eletrocardiografia foram obtidos por meio do eletrocardiograma TEB, versão 219, ECG TC 219, com computador IBM-TEB. Foram calculados os valores (média \pm DP e mediana) do intervalo Qt corrigido (Qtc) de 62 diabéticos e 82 controles. A fórmula utilizada para medida do Qtc foi: intervalo $Q t / \sqrt{ } \mathrm{R}-\mathrm{R}$ anterior na derivação D2 (20).

\section{Análise estatística}

Os dados obtidos neste estudo foram registrados pelo pesquisador e registrados em planilha eletrônica (Microsoft Excel ${ }^{\circledR}$ ) conferidos e cálculados pelo programa Statistica ${ }^{\circledR}$.

As variáveis quantitativas foram representadas em médias \pm DP. Diferenças entre os grupos foram avaliadas por meio do teste $t$ de Student para amostras independentes. Para avaliação de associação entre variáveis quantitativas, estimou-se o coeficiente de correlação linear de Pearson. Para identificação de preditores importantes para variáveis da função diastólica, ajustaram-se modelos de regressão linear múltipla. Valores de $\mathrm{p}<$ 0,05 indicaram significância estatística.

\section{RESULTADOS}

\section{Características gerais}

Os pacientes e controles foram comparados em relação à idade, ao peso, à altura, ao IMC, à SC, ao sexo e à pres- 
são arterial sistêmica, conforme mostrado na tabela 1 . Os diabéticos tinham peso $(\mathrm{p}=0,04), \operatorname{IMC}(\mathrm{p}=0,006) \mathrm{e}$ superfície corpórea $(\mathrm{p}=0,053)$ significativamente maiores que os controles.

Tabela 1. Valores médios \pm DP das variáveis idade, peso, altura, IMC e SC de pacientes portadores de DM1 e controles

\begin{tabular}{lccc}
\hline Variável & $\begin{array}{c}\text { DM1 } \\
(\mathbf{n = 6 7})\end{array}$ & $\begin{array}{c}\text { Controle } \\
(\mathbf{n = 8 4})\end{array}$ & Valor de $\mathbf{p}$ \\
\hline Idade (anos) & $\begin{array}{c}10,34 \pm 3,69 \\
(3-17)\end{array}$ & $\begin{array}{c}9,46 \pm 4,24 \\
(2-17)\end{array}$ & 0,183 \\
& $\begin{array}{c}38,9 \pm 16,6 \\
(13,9-80,3)\end{array}$ & $\begin{array}{c}33,3 \pm 16,31 \\
(11,7-97)\end{array}$ & 0,040 \\
Peso (kg) & $\begin{array}{c}141,61 \pm 20,86 \\
135,99 \pm 23,54\end{array}$ & 0,127 \\
Altura (cm) & $(92,4-172)$ & $(86-187)$ & \\
& $18,41 \pm 3,59$ & $\begin{array}{c}16,87 \pm 3,15 \\
(7,1-27,73)\end{array}$ & 0,006 \\
IMC & $(12,23-27,5)$ & \\
& $1,23 \pm 0,34$ & $1,11 \pm 0,36$ & 0,053 \\
SC & $(0,585-1,92)$ & $(0,539-2,22)$ & \\
& $62,01 \pm 23,66$ & $63,53 \pm 28,6$ & 0,728 \\
Massa VE & $(22,6-115)$ & $(25,9-153)$ & \\
(gramas) & & & \\
\hline
\end{tabular}

Em relação ao sexo, $62 \%$ dos pacientes e $52,4 \%$ dos controles pertenciam ao grupo feminino. Não houve diferença significativa dos valores de pressão arterial entre os grupos. A maioria dos pacientes (65\%) estava na puberdade. Setenta e oito por cento dos diabéticos tinham tempo de doença inferior a cinco anos. Dessa forma, em relação à microalbuminúria, somente $22 \%$ dos portadores de DMl de nossa população dosaram a substância na urina e todos tiveram resultados negativos. Valores de HbAlC foram obtidos de 65 pacientes e estavam acima dos valores recomendáveis em $72 \%$ dos casos. A média de HbAlC na amostra dos portadores de DMl foi de 9,53 $\pm 2,01(5,6-16,65)$. Em relação ao intervalo Qt corrigido, não houve diferença entre pacientes com DMl e controles.

\section{Doppler convencional e tecidual}

As velocidades das ondas $\mathrm{E}(\mathrm{p}=<0,001)$ e $\mathrm{A}(\mathrm{p}=$ 0,037 ) mitral foram menores nos diabéticos, enquanto os valores de TDE e TRIV foram maiores $(p=0,003$ e $\mathrm{p}=0,015$, respectivamente). $\mathrm{O}$ índice de TEI do ventrículo esquerdo foi normal em ambos os grupos (média 0,26 \pm 0,10 nos diabéticos e 0,26 $\pm 0,09$ nos controles).

Quando se analisaram os parâmetros diastólicos por meio do Doppler convencional (onda E e relação E/A) e tecidual (onda E' e relação E'/A'), viu-se que as alte- rações diastólicas foram mais prevalentes no grupo entre 9 e 12 anos. Os demais parâmetros de Doppler convencional e tecidual não foram significativamente diferentes entre os dois grupos, conforme mostra a tabela 2.

Tabela 2. Valores médios \pm DP das variáveis diastólicas na população total de pacientes portadores de DM1 e controles

\begin{tabular}{|c|c|c|c|}
\hline Variável & $\begin{array}{c}\text { DM1 } \\
(\mathrm{N}=67)\end{array}$ & $\begin{array}{c}\text { Controles } \\
(\mathrm{N}=84)\end{array}$ & Valor de $p$ \\
\hline $\mathrm{E}(\mathrm{m} / \mathrm{s})$ & $\begin{array}{l}1,02 \pm 0,18 \\
(0,727-1,58)\end{array}$ & $\begin{array}{l}1,13 \pm 0,15 \\
(0,761-1,44)\end{array}$ & $<0,001$ \\
\hline$A(m / s)$ & $\begin{array}{c}0,61 \pm 0,12 \\
(0,428-1)\end{array}$ & $\begin{array}{c}0,66 \pm 0,13 \\
(0,433-1,127)\end{array}$ & 0,037 \\
\hline$E / A$ & $\begin{array}{c}1,68 \pm 0,30 \\
(1,14-2,5)\end{array}$ & $\begin{array}{c}1,76 \pm 0,31 \\
(1,21-2,5)\end{array}$ & 0,108 \\
\hline TDE (ms) & $\begin{array}{c}210,23 \pm 40,23 \\
(99-316)\end{array}$ & $\begin{array}{c}190,03 \pm 42,09 \\
(103,3-299)\end{array}$ & 0,003 \\
\hline TRIV (ms) & $\begin{array}{c}63,32 \pm 9,19 \\
(44,6-87,6)\end{array}$ & $\begin{array}{c}59,98 \pm 7,45 \\
\quad(44,6-81)\end{array}$ & 0,015 \\
\hline$E^{\prime}(m / s)$ & $\begin{array}{c}0,14 \pm 0,02 \\
(0,088-0,19)\end{array}$ & $\begin{array}{c}0,14 \pm 0,02 \\
(0,103-0,209)\end{array}$ & 0,182 \\
\hline$A^{\prime}(m / s)$ & $\begin{array}{c}0,08 \pm 0,01 \\
(0,051-0,118)\end{array}$ & $\begin{array}{c}0,08 \pm 0,01 \\
(0,054-0,137)\end{array}$ & 0,349 \\
\hline$E^{\prime} / A^{\prime}$ & $\begin{array}{c}1,82 \pm 0,36 \\
(1-2,72)\end{array}$ & $\begin{array}{c}1,92 \pm 0,35 \\
(1,14-3,1)\end{array}$ & 0,099 \\
\hline S Tissular (m/s) & $\begin{array}{l}0,08 \pm 0,01 \\
(0,06-0,113)\end{array}$ & $\begin{array}{c}0,08 \pm 0,01 \\
(0,063-0,138)\end{array}$ & 0,135 \\
\hline $\begin{array}{l}\text { *Veia pulmonar S } \\
(\mathrm{m} / \mathrm{s})\end{array}$ & $\begin{array}{c}0,54 \pm 0,08 \\
(0,414-0,75)\end{array}$ & $\begin{array}{c}0,55 \pm 0,09 \\
(0,281-0,777)\end{array}$ & 0,669 \\
\hline $\begin{array}{l}{ }^{*} \text { Veia pulmonar D } \\
(\mathrm{m} / \mathrm{s})\end{array}$ & $\begin{array}{c}0,64 \pm 0,10 \\
(0,454-0,969)\end{array}$ & $\begin{array}{c}0,689 \pm 0,103 \\
(0,474-0,97)\end{array}$ & 0,221 \\
\hline $\begin{array}{l}{ }^{*} \text { Ar veia } \\
\text { pulmonar }(\mathrm{cm} / \mathrm{s})\end{array}$ & $\begin{array}{l}19,64 \pm 3,85 \\
(11,7-29,26)\end{array}$ & $\begin{array}{c}20,78 \pm 3,32 \\
(14,5-34,4)\end{array}$ & 0,064 \\
\hline $\begin{array}{l}\text { *Veia pulmonar A } \\
\text { duração (ms) }\end{array}$ & $\begin{array}{c}101,50 \pm 21,83 \\
(65-213,5)\end{array}$ & $\begin{array}{c}99,88 \pm 14,76 \\
(49,6-130,3)\end{array}$ & 0,628 \\
\hline
\end{tabular}

* As variáveis veia pulmonar $S$ e $D$, Ar veia pulmonar e veia pulmonar $A$ duração foram coletadas em 56 pacientes devido a variações respiratórias durante 0 exame (solicitada notificação que esses parâmetros foram coletados em apenas 56 pacientes e não nos 67 ).

\section{Diferenças entre os sexos}

Quando a função diastólica foi avaliada segundo o sexo, encontraram-se alterações de parâmetros diastólicos apenas nas meninas. Assim, ondas E mitral e "a" reversa pulmonar foram menores ( $\mathrm{p}=<0,001$ e 0,004 , respectivamente) e TDE $(212,4 \pm 34,62 v s .192,66 \pm 43,91)$ $(\mathrm{p}=0,003)$ e TRIV $(64,69 \pm 8,39$ vs. $58,25 \pm 6,63)$ $(\mathrm{p}=0,015)$ maiores nas diabéticas em relação aos controles; e a faixa etária de 13 a 17 anos foi a que apresentou maior número de alterações diastólicas (onda E mitral, TRIV, onda D da veia pulmonar e onda "a" reversa pulmonar), mostrado na tabela 3 . 
Tabela 3. Valores médios \pm DP da função diastólica no sexo feminino de portadores de DM1 e controles na faixa etária de 13 a 17 anos

\begin{tabular}{lccc}
\hline Variável & DM $\mathbf{1}(\mathbf{N}=\mathbf{1 2})$ & $\begin{array}{c}\text { Controles } \\
(\mathbf{N}=\mathbf{1 7})\end{array}$ & Valor de p \\
\hline $\mathrm{E}(\mathrm{m} / \mathrm{s})$ & $0,95 \pm 0,14$ & $\begin{array}{c}1,08 \pm 0,14 \\
(0,76-1,28)\end{array}$ & 0,027 \\
& $(0,73-1,18)$ & $(0,78)$ & \\
$\mathrm{A}(\mathrm{m} / \mathrm{s})$ & $0,54 \pm 0,07$ & $0,57 \pm 0,13$ & 0,440 \\
& $(0,46-0,68)$ & $(0,43-0,99)$ & \\
E/A & $1,80 \pm 0,30$ & $1,92 \pm 0,31$ & 0,287 \\
& $(1,21-2,18)$ & $(1,29-2,49)$ & \\
TDE (ms) & $230,12 \pm 39,1$ & $210,72 \pm 44,38$ & 0,235 \\
& $(168,7-301,6)$ & $(153-297)$ & \\
TRIV (ms) & $68,31 \pm 5,32$ & $59,72 \pm 5,25$ & $<0,001$ \\
& $(58,60-76,70)$ & $(51,70-67,30)$ & \\
* Veia pulmonar S & $0,53 \pm 0,05$ & $0,55 \pm 0,09$ & 0,397 \\
(m/s) & $(0,45-0,61)$ & $(0,39-0,69)$ & \\
* Veia pulmonar D & $0,57 \pm 0,08$ & $0,67 \pm 0,11$ & 0,019 \\
(m/s) & $(0,45-0,74)$ & $(0,49-0,92)$ & \\
* Ar veia pulmonar & $17,16 \pm 2,77$ & $21,47 \pm 2,37$ & $<0,001$ \\
(cm/s) & $(11,83-23,30)$ & $(16,5-25,8)$ & \\
* Veia pulmonar A & $107,45 \pm 13,26$ & $99,79 \pm 12,92$ & 0,131 \\
duração (ms) & $(87,50-135)$ & $(76,30-119)$ & \\
\hline
\end{tabular}

*As varíáveis veia pulmonar $S$ e D, ar veia pulmonar e veia pulmonar A duração foram coletadas em 56 pacientes devido a variações respiratórias durante 0 exame (solicitada notificação que esses parâmetros foram coletados em apenas 56 pacientes e não nos 67).

\section{Correlação de Pearson e regressão múltipla}

A tabela 4 mostra que o IMC dos diabéticos teve correlação positiva com as variáveis TDE e TRIV. No modelo de regressão múltipla para avaliação da influência conjunta das variáveis idade, tempo de duração de doença, IMC e $\mathrm{HbAlC}$, sobre as variáveis ondas $\mathrm{E}$ e A mitral, TDE e TRIV, somente a onda A mitral sofreu influência da idade e tempo de diabetes (beta $=$ $-0,577, \mathrm{p}=0,004$ e beta $=0,346$ e $\mathrm{p}=0,033$, respectivamente).

Tabela 4 Correlação de Pearson entre variáveis diastólicas e IMC e variáveis bioquímicas de pacientes portadores de DM1

\begin{tabular}{|c|c|c|}
\hline Variável & IMC & HBA1C \\
\hline \multirow[t]{2}{*}{$\mathrm{E}(\mathrm{m} / \mathrm{s})$} & 0,00 & $-0,23$ \\
\hline & $p=0,984$ & $p=0,140$ \\
\hline \multirow[t]{2}{*}{$\mathrm{A}(\mathrm{m} / \mathrm{s})$} & $-0,21$ & $-0,06$ \\
\hline & $p=0,175$ & $p=0,682$ \\
\hline \multirow[t]{2}{*}{ TDE (ms) } & 0,33 & 0,09 \\
\hline & $p=0,028$ & $p=0,576$ \\
\hline \multirow[t]{2}{*}{ TRIV (ms) } & 0,33 & 0,25 \\
\hline & $p=0,028$ & $p=0,096$ \\
\hline
\end{tabular}

\section{DISCUSSÃO}

Alterações cardiovasculares do diabetes melito são observadas em um estágio tardio da doença. Porém, quando elas se iniciam? A infância poderia ser o estágio subclínico inicial?. Se presente em crianças, quais os fatores que influenciariam seu aparecimento?

No estudo realizado, foram encontrados os seguintes resultados: 1 . Diferenças de parâmetros diastólicos do VE com maior prevalência na faixa etária dos pacientes de 9 a 12 anos. 2. O sexo feminino teve o maior número de alterações de parâmetros diastólicos do VE. 3. A faixa etária do sexo feminino com maior prevalência de alterações nos parâmetros de VE foi entre $13 \mathrm{e}$ 17 anos. 4. Os valores de IMC apresentaram correlação positiva com os valores de TRIV e TDE. 5. O tempo de doença foi um fator preditivo positivo para aumento de velocidade da onda A mitral. 6. As alterações incipientes dos parâmetros diastólicos de VE podem sugerir disfunção diastólica.

A disfunção diastólica no DM pode ser causada por rigidez da parede do $\mathrm{VE}$, deposição dos produtos finais de glicosilação avançada, fibrose cardíaca, e complicações diabéticas $(5,6)$. Estudos anteriores descreveram alterações diastólicas em portadores de DMl na faixa etária pediátrica que apresentavam fração de ejeção normal $(12,21)$. Adal e cols. (22) avaliaram portadores de DMl com média de idade de $12 \pm 4,2$ anos e encontraram alterações da onda A mitral, relação E/A e TRIV. No estudo de Salem e cols. (2l), portadores de DMl jovens tiveram o fragmento $\mathrm{N}$-terminal do pró-peptídeo natriurético cerebral significamente elevado e este se correlacionou fortemente com a disfunção diastólica de VE isolada.

Em relação ao sexo, a função diastólica de nossas pacientes é concordante com o estudo de Suys e cols. (12), que afirmou que meninas diabéticas púberes com IMC maior que os controles apresentavam anormalidades de enchimento diastólico ao Doppler convencional e tecidual.

Outro dado evidenciado em nosso estudo foi que o IMC elevado nos portadores de DMl se correlacionou positivamente com o TDE e TRIV ( $\mathrm{p}=0,028$ ) em ambos os sexos. Embora o pareamento caso e controle para IMC não tenha sido realizado, a comparação dos dados foi validada pelo fato de que a massa ventricular esquerda era semelhante entre os grupos.

$\mathrm{O}$ valor de TDE significativamente maior nos diabéticos significou tempo de enchimento ventricular esquerdo mais lento e, possivelmente, disfunção diastólica, como sugerido por Horowitz (23). Corroborando 
o achado de TDE aumentado, encontrou-se tempo de relaxamento isovolumétrico (TRIV) aumentado, o qual indica rigidez da parede miocárdica. Dados semelhantes foram reportados por Tamuri e cols. (24) em diabéticos adultos e por Adal e cols. (22) em pacientes pediátricos.

Em relação a NAC e sua contribuição nas alterações cardiovasculares do DM, nosso estudo a excluiu pelos valores normais do intervalo QT que, de acordo com Silvieri e cols. (25) e Pappachan e cols. (26), é um método com razoável sensibilidade, especificidade e valor preditivo positivo para diagnóstico de NAC.

Outro dado relevante no presente estudo foi o maior número de alterações diastólicas nos pacientes com maior tempo de doença ( 5 anos, $\mathrm{n}=15$ ). Essa associação é concordante com estudos anteriores realizados por Adal e cols. (22), Cerrutti e cols. (27) e Lo e cols. (28), entretanto, não foram confirmados por Riggs e Transue (11).

Quanto à análise de regressão múltipla, os valores da onda A mitral foram influenciados pelas variáveis tempo de doença e idade cronológica. Sabendo-se que a onda A mitral na Dopplerecocardiografia traduz a contração atrial, nosso estudo sugere que, com o aumento da idade cronológica e tempo de doença, o miocárdio dos portadores de DMl fique mais rígido, necessitando de maior contribuição atrial para o enchimento de VE. Dados semelhantes foram relatados por Suys e cols. (12).

Em conclusão, o presente estudo evidenciou alterações de parâmetros diastólicos em pacientes portadores de $\mathrm{DMl}$ na faixa etária pediátrica e principalmente em meninas púberes com IMC mais elevado. Não houve correlação da função diastólica com a hemoglobina glicada Alc, porém, tempo de doença e idade cronológica podem influenciar em parâmetros diastólicos. Esses dados podem sugerir que o início da miocardiopatia diabética pode ser subclínico e começar na infầncia.

Agradecimentos: a todos os pacientes, pais e responsáveis que se dispuseram a colaborar com este estudo. À Unidade de Endocrinologia Pediátrica da Universidade Federal do Paraná por toda a gentileza, humanidade, carisma e sabedoria, representada pela figura do meu orientador Dr. Luiz de Lacerda Filho.

Declaração: os autores declaram não haver conflitos de interesse científico neste estudo.

\section{REFERÊNCIAS}

1. Defronzo RA. International textbook of diabetes mellitus. 3rd edition. Chichester, West Sussex, Hoboken, NJ: John Wiley; 2004.
2. Kannel WB, Hjortland M, Castelli WP. Role of diabetes in congestive heart failure: the Framingham study. Am J Cardiol. 1974;34(1):29-34.

3. Rubler S, Dluglash J,YuceogluYZ, KumralT, Branwood AW, Grishman A. New type of cardiomyopathy associated with diabetic glomerulosclerosis. Am J Cardiol. 1972;30(6):595-602.

4. Codinach-Huix P, Freixa-Pamias R. Diabetic cardiomyopathy: concept, heart function, and pathogenesis. An Med Interna. 2002;19(6):313-20.

5. Okoshi K, Guimarães JFC, Muzio BP, Fernandes AAH, Okoshi MP. Miocardiopatia diabética. Arq Bras Endocrinol Metab. 2007;51:160-7.

6. Shapiro LM. Echocardiographic features of impaired ventricular function in diabetes mellitus. Br Heart J. 1982;47(5):439-44.

7. Zarich SW, Arbuckle BE, Cohen LR, Roberts M, Nesto RW. Diastolic abnormalities in young asymptomatic diabetic patients assessed by pulsed Doppler echocardiography. J Am Coll Cardiol. 1988;12:114-20.

8. Huikuri HV, Airaksinen JKE, Ljlia M, Takkunuen JT. Echocardiographic evaluation of left ventricular response to isometric exercise in young insulin-dependent diabetics. Acta Diabetol Lat. 1986;23(3):193-200.

9. Raev DC. Which left ventricular function is impaired earlier in the evolution of diabetic cardiomyopathy? An echocardiographic study of young type I diabetic patients. Diabetes Care. 1994;17(7):633-9.

10. Hausdorf G, Rieger U, Koepp P. Cardiomyopathy in childhood diabetes mellitus: incidence, time of onset, and relation to metabolic control. Int J Cardiol (Netherlands). 1988;19:225-36.

11. Riggs TW, Transue D. Doppler echocardiographic evaluation of left ventricular diastolic function in adolescents with diabetes mellitus. Am J Cardiol. 1990;65:899-902.

12. Suys BE, Katier N, Rooman RPA, Matthys R, Beeck LO, Caju MVL, et al. Female children and adolescents with type 1 diabetes have more pronounced early echocardiographic signs of diabetic cardiomyopathy. Diabetes Care. 2004;27:1947-53.

13. Karamitsos TD, Karvounis HI, Dalamanga EG, Papadopoulos CE, Didangellos TP, Karamitsos DT, et al. Early diastolic impairment of diabetic heart: the significance of right ventricle. Int $\mathrm{J}$ Cardiol. 2007;114:218-23.

14. Marshall WA, Tanner JM. Variations in the pattern of pubertal changes in boys. Arch Dis Child. 1970;45:13-23.

15. Fonseca V, Blonde L, Gerstein CH, Hirsc I, Kahn S, Korytkowski TM. Standards of medical care in diabetes -2007 . Diabetes Care. 2007;30(Suppl 1):S4-41.

16. Daniels RS, Greer RF. Lipid screening and cardiovascular health in childhood, and the committee on nutrition. Pediatrics. 2008;122(1):198-208.

17. Sahn DJ, DeMaria A, Kisslo J, Weyman A. The Committee on M-mode Standardization of the American Society of Echocardiography: recommendations regarding quantitation on M-mode echocardiography: result of a survey of echocardiographic measurements. Circulation. 1978;58:1072-83.

18. Henry WL, Demaria A, Gramiak R, King DL, Kisslo JA, Popp RL. Report of the American Society of Echocardiography Committee on Nomenclature and Standards in Two-dimensional Echocardiography. Circulation. 1980;62:212-7.

19. O'Leary PW. Pediatric diastology: use and limitation of Doppler echocardiography in the evaluation of ventricular diastolic function in children. Prog Pediatr Cardiol. 1999;10:83-93.

20. Bazett HC. An analysis of the time-relations of electrocardiograms. Heart. 1920;7:353-70.

21. Salem M, El Behery S, Adly A, Khalil D, El Hadidi E. Early predictors of myocardial disease in children and adolescents with type 1 diabetes mellitus. Pediatr Diabetes. 2009;10:513-21.

22. Adal E, Koyuncu G, Aydin A, Celebi A, Kavunoglu G, Cam H. Asymptomatic cardiomyopathy in children and adolescents with type 1 diabetes mellitus: association of echocardiographic indi- 
cators with duration of diabetes mellitus and metabolic parameters. J Pediatr Endocrinol Metabol. 2006;19(5):713-26.

23. Horowitz ESK. Estudo ecocardiográfico da função diastólica em crianças. Rev Bras Ecocardiogr. 2002;3:22-32.

24. Tamuri N, Iwasaka T, Takahashi N, Sugiura T, Morita Y, Sumimoto $\mathrm{T}$, et al. Left ventricular diastolic filling properties in diabetic patients during isometric exercise. Cardiology. 1993;83:316-23.

25. Silvieri R, Veglio M, Chinaglia A, Scaglione P, Cavallo-Perin P. Italian Society for the Study of Diabetes, Neuropathy Study Group, Torino. Prevalence of QT prolongation in a type I diabetic population and its association with autonomic neuropathy. Diabet Med. 1993;10:920-4.
26. Pappachan JM, Sebastian J, Bino BC, Jayaprakash K, Vijayakumar K, Sujathan P, et al. Cardiac autonomic neuropathy in diabetes mellitus: prevalence, risk factors and utility of corrected OT interval in the ECG for its diagnosis. Postgrad Med J. 2008;84:205-10.

27. Cerrutti F, Vigo A, Sacchetti C, Bessone A, Barattia G, Morello M, et al. Evaluation of left ventricular diastolic function in insulin dependent diabetic children by M-mode and Doppler echocardiography. Panminerva Med. 1994;36:109-14.

28. Lo SSS, Leslie RDG, Sutton MSJ. Effects of type 1 diabetes mellitus on cardiac function; a study of monozygotic twins. Br Heart J. 1995;73:450-5. 